\title{
The Motion of a Rigid Body with Irrational Natural Frequency
}

\author{
A. I. Ismail $\mathbb{D}^{1,2}$ \\ ${ }^{1}$ Mechanical Engineering Department, College of Engineering and Islamic Architecture, Umm Al-Qura University, Makkah, Saudi \\ Arabia P. O. Box 5555 \\ ${ }^{2}$ Mathematics Department, Faculty of Science, Tanta University, Tanta, P.O. Box 31527, Egypt
}

Correspondence should be addressed to A. I. Ismail; aiismail@uqu.edu.sa

Received 29 August 2020; Accepted 23 November 2020; Published 7 December 2020

Academic Editor: Ivan Giorgio

Copyright (c) 2020 A. I. Ismail. This is an open access article distributed under the Creative Commons Attribution License, which permits unrestricted use, distribution, and reproduction in any medium, provided the original work is properly cited.

In this paper, we consider the problem of the rotational motion of a rigid body with an irrational value of the frequency $\omega$. The equations of motion are derived and reduced to a quasilinear autonomous system. Such system is reduced to a generating one. We assume a large parameter $\mu$ proportional inversely with a sufficiently small component $r_{\mathrm{o}}$ of the angular velocity which is assumed around the major or the minor axis of the ellipsoid of inertia. Then, the large parameter technique is used to construct the periodic solutions for such cases. The geometric interpretation of the motion is obtained to describe the orientation of the body in terms of Euler's angles. Using the digital fourth-order Runge-Kutta method, we determine the digital solutions of the obtained system. The phase diagram procedure is applied to study the stability of the attained solutions. A comparison between the considered numerical and analytical solutions is introduced to show the validity of the presented techniques and solutions.

\section{Introduction}

The rigid body problem of mass $M$ that rotates about a fixed point $O$ is classified according to the natural frequency value in either the uniform gravity field of $g$ acceleration or a Newtonian force one. The case of the rational value of the natural frequency is studied in [1] for a rotating heavy solid about a fixed point with a small velocity about one of the axes of the ellipsoid of inertia. The solutions obtained contain singular cases of the natural frequencies named when $\omega=1,2$, $3,1 / 2,1 / 3, \cdots$. The singular cases that appeared for the natural frequency values like $\omega=1$ (the disk case) and $\omega=0.5$ are considered in $[2,3]$, respectively. It remains for us to study four other cases until the solution of the problem is completed up to the third approximation for any natural frequency of movement. Such cases are classified according to the natural frequency values named; the state of irrational values of the natural frequencies which is the subject of this article besides three singular cases will be studied in the future in shaa Allah classified when $\omega=2,3,1 / 3$.

Let the frameOxyzbe fixed in the body and the frameOXYZbe fixed in space. Assume that $A, B$, and $C$ represent the moments of inertia of the body in the moving coor- dinate system. Suppose $\left(x_{0}, y_{0}, z_{0}\right)$ is the mass center of the body. In the case when $\omega$ is irrational, the equations of motion are obtained and reduced to the following system:

$$
\begin{gathered}
\ddot{x}_{1}+\omega^{2} x_{1}=\mu^{-2} F\left(x_{1}, x_{2}, \dot{x}_{1}, \dot{x}_{2}, \mu^{-1}\right), \\
\ddot{x}_{2}+x_{2}=\mu^{-2} \Phi\left(x_{1}, x_{2}, \dot{x}_{1}, \dot{x}_{2}, \mu^{-1}\right),
\end{gathered}
$$

where

$$
\begin{gathered}
F=\sum_{k=2}^{\infty} \mu^{2-k} F_{k},(F \Phi), \\
\dot{x}_{i}=\frac{d x_{i}}{d \tau}, \quad \tau=\frac{t}{r_{\mathrm{o}}}, \\
x_{1}=x_{1}(p, \gamma),\left(x_{1} x_{2}\right), \\
\mu=c \sqrt{\gamma_{\mathrm{o}}^{\prime \prime}} / r_{0}, \quad 0 \prec \gamma_{\mathrm{o}}^{\prime \prime} \prec 1,
\end{gathered}
$$

where symbols like (ab) mean cyclic permutations and indicate equations which are omitted; $p$ and $\gamma$ are the $x$ 


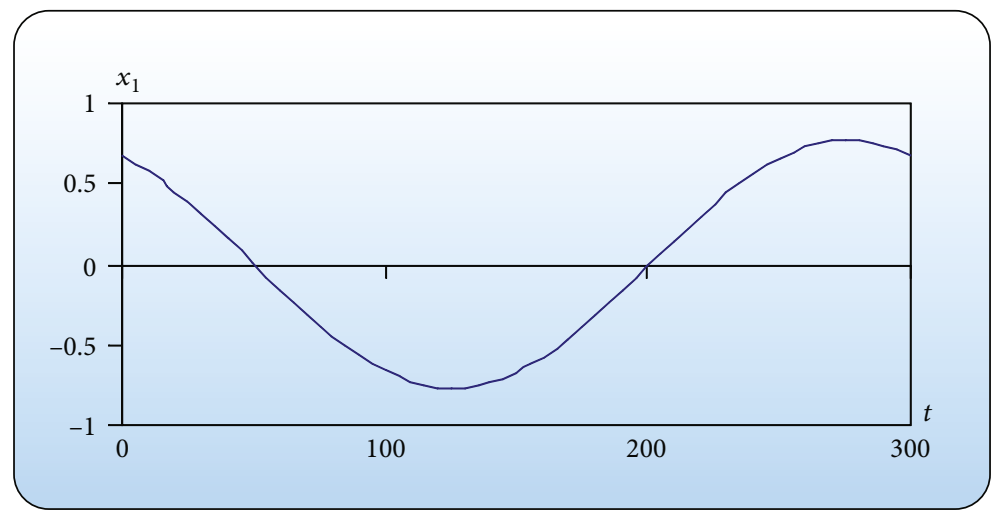

Figure 1: The analytical solution $x_{1}$ against the time $t$.

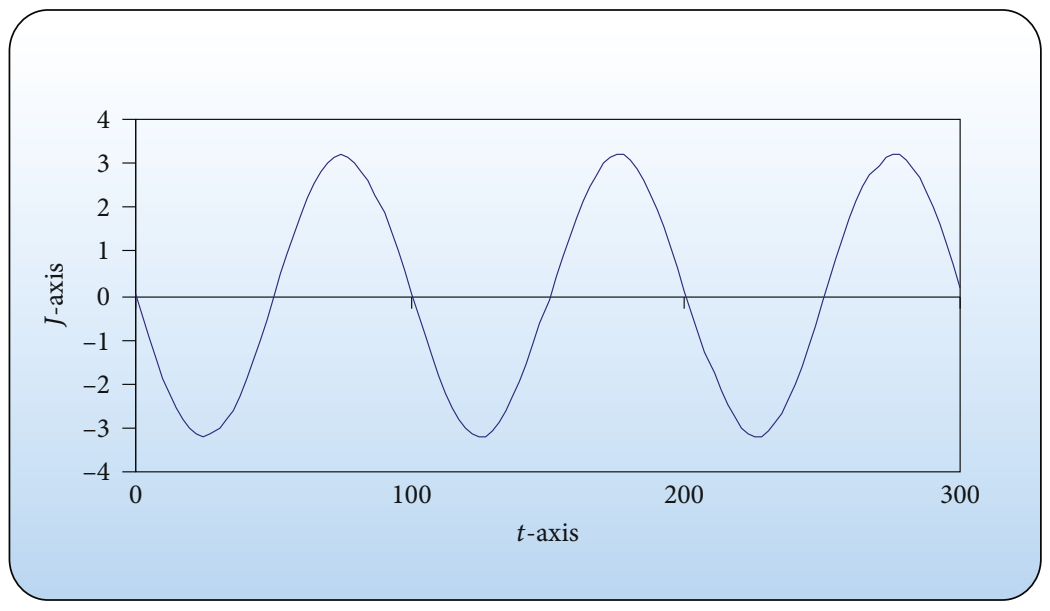

Figure 2: The analytical solution $J=x_{2}$ against the time $t$.

-components of the angular velocity vector and the fixed unit vector in space, respectively; and $c$ is a constant that depends on the rigid body parameters. The system (1) has the following first integral:

$$
x_{2}^{2}+\dot{x}_{2}^{2}+2 \mu^{-1}\left(v x_{1} x_{2}+v_{1} \dot{x}_{1} \dot{x}_{2}+s_{21}\right)+\mu^{-2}(\cdots)=L
$$

where $v, v_{1}$, and $L$ are constants that depend on the rigid body parameters, and the function $s_{21}=s_{21}\left(\tau, \mu^{-1}\right)$ satisfies the condition $s_{21}\left(0, \mu^{-1}\right)=0$ when $\mu \rightarrow \infty$.

\section{The Periodic Solutions}

In this section, we obtain the generating system of (1), and then, we will solve it under a new condition of motion. We adapt a large parameter method [1] to solve the system (1) in the presence of the first integral (4). By putting $\mu \rightarrow \infty$ into the system (1), we get the generating system as in [4] which has generating periodic solutions of a period $T_{0}=2 \pi$.

In this case, from the system (1), the required periodic solutions with a period $\left(T_{0}+\alpha\right)$ are assumed in the following forms [5]:

$$
\begin{gathered}
x_{1}\left(\tau, \mu^{-1}\right)=\eta_{1}\left(\beta_{3}, \mu^{-1}\right) \cos \omega \tau+\eta_{2}\left(\beta_{3}, \mu^{-1}\right) \sin \omega \tau+\sum_{k=2}^{\infty} \mu^{-k} G_{k}(\tau), \\
x_{2}\left(\tau, \mu^{-1}\right)=\left(M_{3}+\beta_{3}\right) \cos \tau+\sum_{k=2}^{\infty} \mu^{-k} H_{k}(\tau),
\end{gathered}
$$

where $M_{3}$ is an arbitrary constant and $\beta_{3}=\beta_{3}\left(\mu^{-1}\right)$ and $\eta_{i}\left(\beta_{3}, \mu^{-1}\right)(i=1,2)$ are analytic functions of $\mu^{-1}$ which vanish when $\mu \rightarrow \infty$. The function $\alpha$ is an analytic function of $\mu^{-1}$ which vanishes when $\mu \rightarrow \infty$. The functions $G_{k}(\tau)$ and $H_{k}($ $\tau$ ) are obtained by substituting (5) into (1) and equating coefficients of like powers of $\mu^{-1}$.

The functions $\eta_{i}\left(\beta_{3}, \mu^{-1}\right)$ can be written in an expansion series as follows [6]:

$$
\eta_{i}\left(\beta_{3}, \mu^{-1}\right)=\sum_{k=1}^{\infty}\left(Q_{k}^{(i)}+\frac{\partial Q_{k}^{(i)}}{\partial M_{3}} \beta_{3}+\frac{1}{2} \frac{\partial^{2} Q_{k}^{(i)}}{\partial M_{3}^{2}} \beta_{3}^{2}+\cdots\right) \mu^{-k}
$$




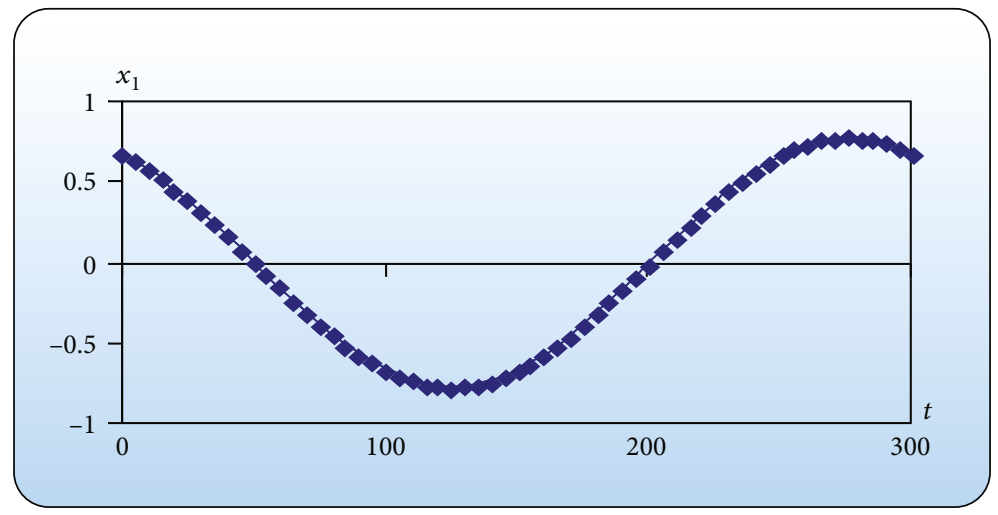

Figure 3: The numerical solution $x_{1}$ against the time $t$.

Using Equation (3), the periodicity conditions are obtained as follows [7]:

$$
\begin{gathered}
x_{1}\left(T_{0}, \mu^{-1}\right)-\eta_{1}\left(\beta_{3}, \mu^{-1}\right)+\alpha \omega \eta_{2}\left(\beta_{3}, \mu^{-1}\right)=0, \\
\dot{x}_{1}\left(T_{0}, \mu^{-1}\right)-\omega \eta_{2}\left(\beta_{3}, \mu^{-1}\right)-\alpha \omega^{2} \eta_{1}\left(\beta_{3}, \mu^{-1}\right)=0, \\
\dot{x}_{2}\left(T_{0}, \mu^{-1}\right)-\alpha\left(M_{3}+\beta_{3}\right)=0 .
\end{gathered}
$$

Under condition (7) and series (6), it produces an endless system of equations that designates the coefficients $Q_{k}^{(i)}$. The functions $G_{k}(\tau)$ and $H_{k}(\tau)$ are expressed as in [8].

Now, we aim to find the periodic solutions of the generalized system. According to [9], series (6) begins from a term of order not lower than $\mu^{-2}$. It follows that the expansion of $x_{1}\left(\tau, \mu^{-1}\right)$ represents a power series that begins from a term of order not lower than $\mu^{-2}$. In this case, the quantities $H_{2}($ $\left.T_{0}\right)$ and $\dot{H}_{2}\left(T_{0}\right)$ are obtained.

Introduce the following variables [10]:

$$
\begin{gathered}
x_{1}=p c^{-1} \sqrt{\gamma_{0}^{\prime \prime}}-\mu^{-1}\left(e+e_{1} x_{2}\right), x_{2}=\gamma\left(\gamma_{0}^{\prime \prime}\right)^{-1}-\mu^{-1} v x_{1}, \\
q c^{-1} \sqrt{\gamma_{0}^{\prime \prime}}=-A_{1}^{-1} \dot{x}_{1}+\mu^{-1} A_{1}^{-1}\left(y_{0}^{\prime} a^{-1}-e_{2} \dot{x}_{2}\right)+\cdots \\
r r_{0}^{-1}=1+0.5 \mu^{-2} s_{11}+\cdots, \gamma^{\prime}\left(\gamma_{0}^{\prime \prime}\right)^{-1}=\dot{x}_{2}+\mu^{-1} v_{1} \dot{x}_{1}+\cdots, \\
\gamma^{\prime \prime}\left(\gamma_{0}^{\prime \prime}\right)^{-1}=1+\mu^{-1} s_{21}+\mu^{-2}\left(s_{22}-0.5 s_{11}\right)+\cdots,
\end{gathered}
$$

where $e_{1}=\left(z_{0}^{\prime} /\left(1-\omega^{2}\right)\right)\left(A_{1} b^{-1}-a^{-1}\right), e_{2}-e_{1}=z_{0}^{\prime} a^{-1}$, and $y_{o}{ }^{\prime}, z_{o}{ }^{\prime}, a, b, A_{1}$ are constants that depend on the rigid body parameters. The vectors $[p, q, r]$ and $\left[\gamma, \gamma^{\prime}, \gamma^{\prime \prime}\right]$ represent the components of the angular velocity and the unit vector of the downward fixed axis in space, respectively, and $s_{i i}=$ $s_{i i}\left(\tau, \mu^{-1}\right), i=1,2$, satisfies the condition $s_{i i}\left(0, \mu^{-1}\right)=0$ when $\mu \rightarrow \infty$.

Let $r_{0}$ be sufficiently small; we define a large parameter $\mu$ and apply the large parameter method [3] to obtain the analytical solutions in the form of power series expansions in terms of $\mu^{-1}$ as follows:

$$
\begin{gathered}
p c^{-1} \sqrt{\gamma_{0}^{\prime \prime}}=\mu^{-1}\left[\frac{x_{0}^{\prime}}{1-a} \gamma_{0}^{\prime \prime}+e_{1}\left(M_{3}+\beta_{3}\right) \cos \tau\right]+\cdots, \\
q c^{-1} \sqrt{\gamma_{0}^{\prime \prime}}=\mu^{-1}\left[\mu^{-1} \frac{y_{0}^{\prime}}{1-b} \gamma_{0}^{\prime \prime}+e_{2} A_{1}^{-1}\left(M_{3}+\beta_{3}\right) \sin \tau\right]+\cdots, \\
r r_{0}^{-1}=1-\mu^{-2}\left(M_{3}+\beta_{3}\right)\left[x_{0}^{\prime}(1-\cos \tau)+y_{0}^{\prime} \sin \tau\right]+\cdots, \\
\gamma=\left(M_{3}+\beta_{3}\right) \cos \tau-0.5 \mu^{-2} \Gamma^{2} \cos \tau+\cdots, \\
\gamma^{\prime}=-\left(M_{3}+\beta_{3}\right) \sin \tau+0.5 \mu^{-2} \Gamma^{2} \sin \tau+\cdots, \\
\gamma^{\prime \prime}=\gamma_{0}^{\prime \prime}+\mu^{-2}\left(M_{3}+\beta_{3}\right)\left[\frac{x_{0}^{\prime}}{1-a} \gamma_{0}^{\prime \prime}(1-\cos \tau)+\frac{y_{0}^{\prime}}{1-b} \gamma_{0}^{\prime \prime} \sin \tau\right. \\
\left.-0.5\left(M_{3}+\beta_{3}\right) \frac{C_{1} z_{0}^{\prime}}{a+b-1}(1-\cos 2 \tau)\right]+\cdots,
\end{gathered}
$$

where $C_{1}, x_{0}{ }^{\prime}$ are constants that depend on the rigid body parameters, and

$$
0<\Gamma<1
$$

The correction of the period $\alpha$ is obtained in the form

$$
\alpha=2 \mu^{-2} \pi n\left[\left(M_{3}+\beta_{3}\right) x_{0}^{\prime}-z_{0}^{\prime} \gamma_{0}^{\prime \prime}\right]+\cdots
$$

\section{The Geometric Interpretation of Motion}

In this section, we discuss the problem geometrically to show the orientation of the body at any instant in time. Substituting Equation (9) into Euler's angles $\psi, \theta$, and $\varphi$ in which $t$ has been replaced by $t+h$, using $M_{3}=\tan \theta_{o}$, we obtain the 


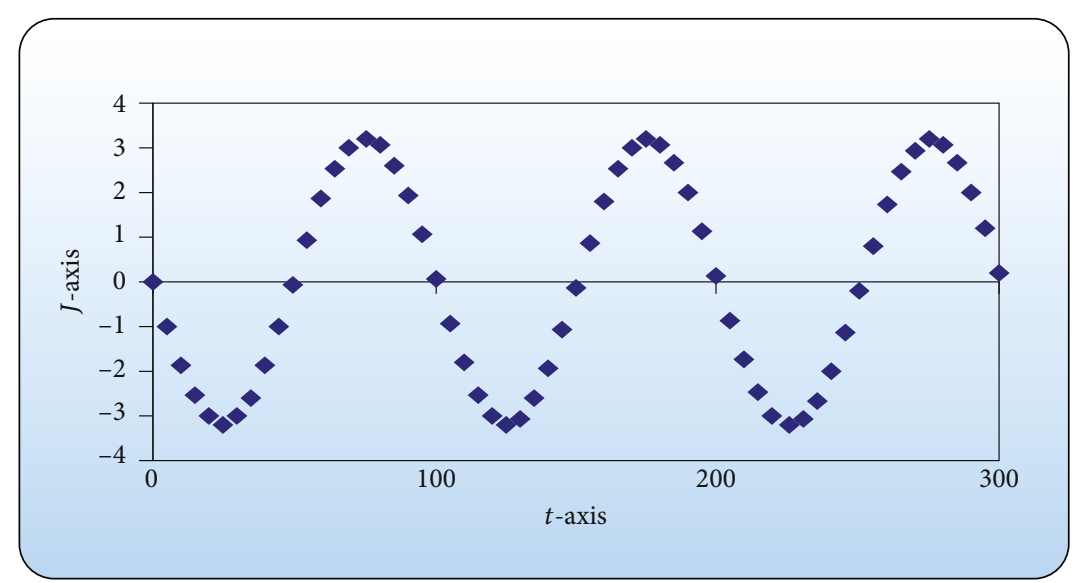

FIgURE 4: The numerical solution $J=x_{2}$ against the time $t$.

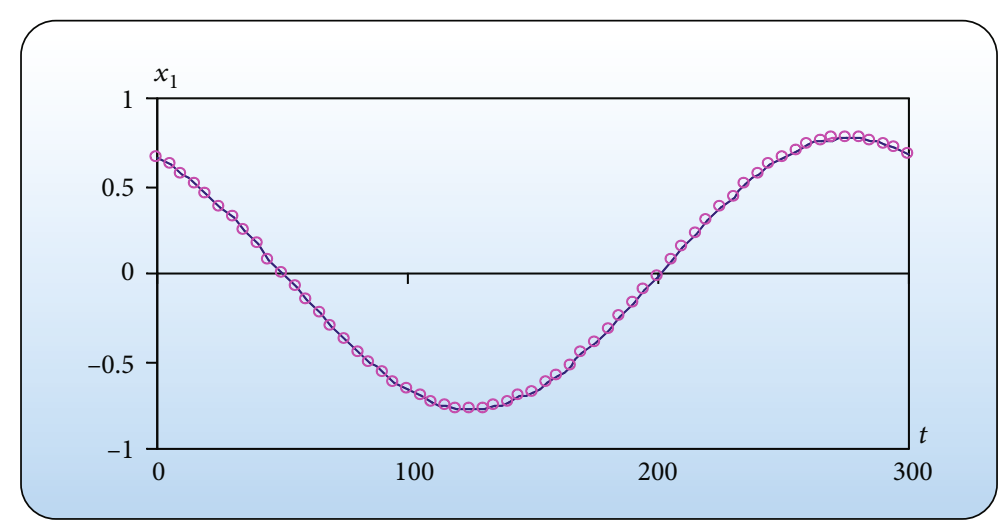

FIgURE 5: The analytical and numerical solutions $x_{1}$ against the time $t$.

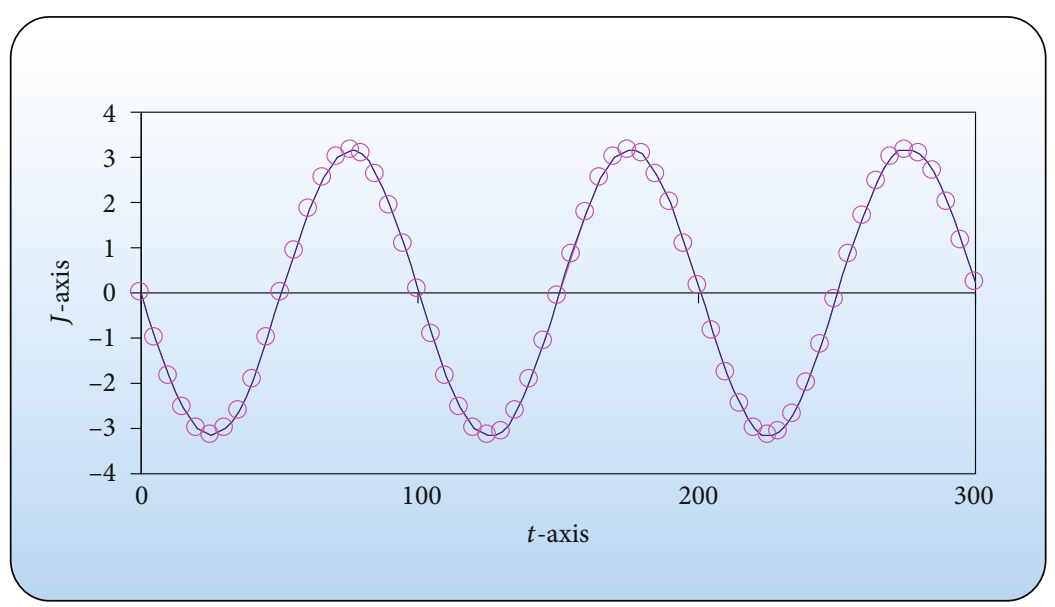

FIgURE 6: The analytical and numerical solutions $J=x_{2}$ against the time $t$. 


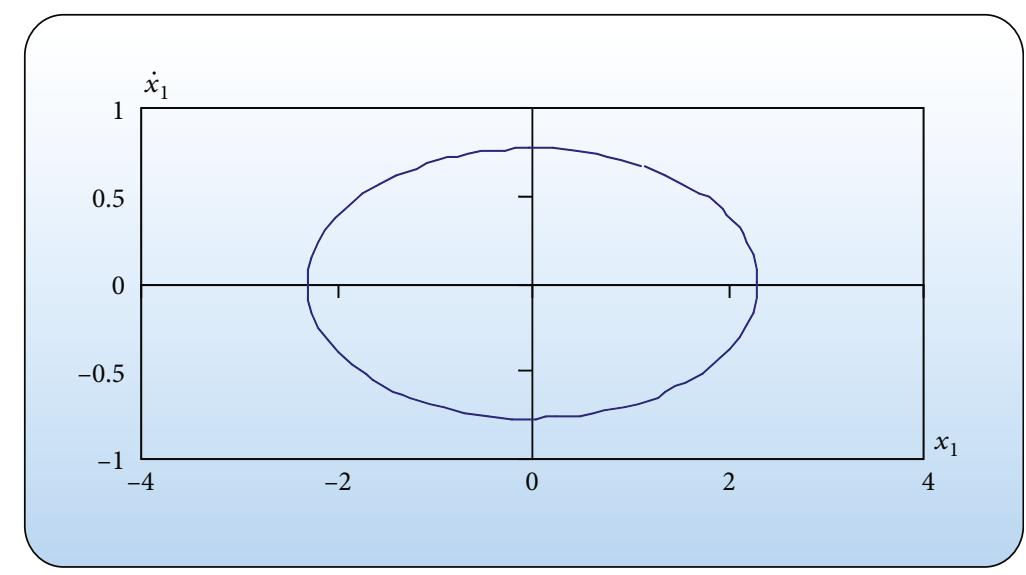

FIgURE 7: The stability of the analytical solution $\dot{x}_{1} \equiv x_{1}$.

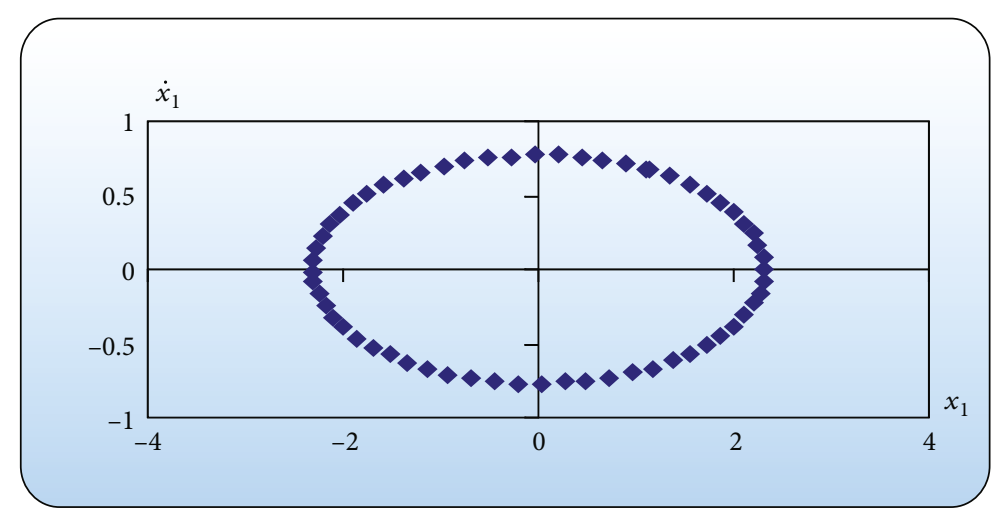

FIgURE 8: The stability of the numerical solution $\dot{x}_{1} \equiv x_{1}$.

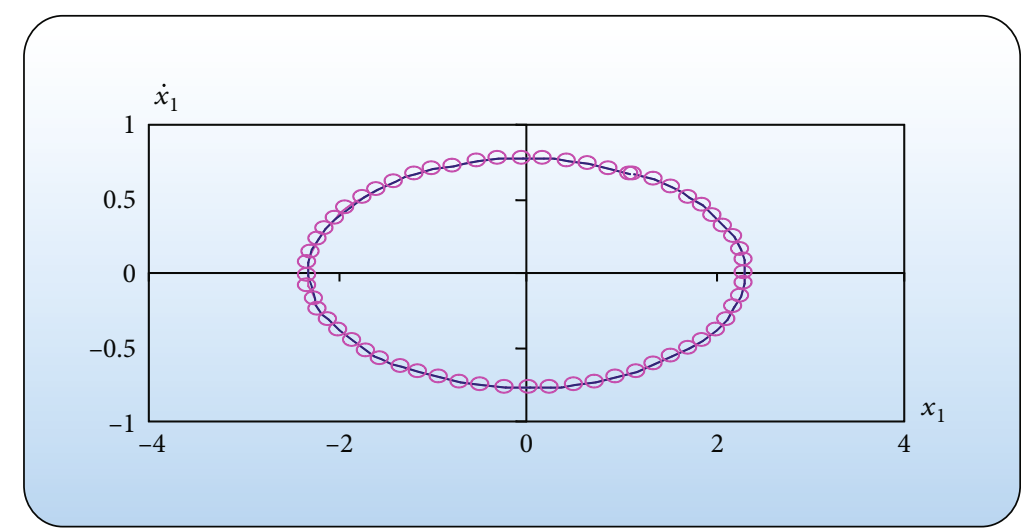

Figure 9: The stability of the analytical and numerical solutions $\dot{x}_{1} \equiv x_{1}$.

following expressions for the angles [11]:

$$
\begin{gathered}
\theta=\theta_{o}-\mu^{-2}\left[\theta^{(1)}(t+h)-\theta^{(1)}(h)\right]+\cdots, \\
\psi=\psi_{o}-M g\left(C r_{o}\right)^{-1} z_{o} t+\mu^{-1} \operatorname{cosec} \theta_{o} \sqrt{\cos \theta_{o}} \\
\cdot\left[\psi^{(1)}(t+h)-\psi^{(1)}(h)\right]+\cdots,
\end{gathered}
$$$$
\varphi=\varphi_{o}+\left[r_{o}+M g\left(C r_{o}\right)^{-1}\left(z_{o} \cos \theta_{o}-x_{o} \sin \theta_{o}\right)\right] t+\cdots,
$$

where

$$
\begin{aligned}
\theta^{(1)}(t)= & y_{o}{ }^{\prime} a^{-1} A_{1}{ }^{-1} \sin r_{o}{ }^{-1} t+x_{o}{ }^{\prime} b^{-1} B_{1}{ }^{-1} \cos r_{o}{ }^{-1} t \\
& -0.5 z_{o}{ }^{\prime} \tan \theta_{o}\left(A_{1}-1\right)\left(A_{1}+1\right)^{-1} \cos 2 r_{o}{ }^{-1} t \\
\psi^{(1)}(t)= & y_{o}{ }^{\prime} a^{-1} A_{1}{ }^{-1} \cos r_{o}{ }^{-1} t-x_{o}{ }^{\prime} b^{-1} B_{1}^{-1} \sin r_{o}{ }^{-1} t \\
& +0.25 \tan \theta_{o}\left(e_{1}+e_{2} / A_{1}\right) \sin 2 r_{o}{ }^{-1} t
\end{aligned}
$$

where $B_{1}$ is a constant depending on the moments of inertia. 


\section{Numerical Considerations}

In this section, we study the analytical and numerical solutions for our problem as follows.

4.1. The Analytical Solutions. We rewrite the analytical solutions $x_{1}, x_{2}$ in the following form:

$$
\begin{aligned}
& x_{1}\left(1-\mu^{-2} e_{1} v\right)=-\mu^{-1}\left\{e+\frac{x_{o}{ }^{\prime} \gamma_{o}^{\prime \prime}}{1-a}+e_{1}\left(M_{3}+\beta_{3}\right) \cos r_{o}^{-1} t\right. \\
& -e_{1}\left(\gamma_{o}^{\prime \prime}\right)^{-1}\left[\left(M_{3}+\beta_{3}\right) \cos r_{o}^{-1} t\right. \\
& \left.\left.-0.5 \mu^{-2} \Gamma^{2} \cos r_{o}^{-1} t\right]\right\}+\cdots \\
& \dot{x}_{1}\left(1-\mu^{-2} e_{1} v\right)=-\mu^{-1}\left\{-e_{1}\left(M_{3}+\beta_{3}\right) \sin r_{o}^{-1} t+e_{1}\left(\gamma_{o}{ }^{\prime \prime}\right)^{-1}\right. \\
& \text { - } \left.\left[\left(M_{3}+\beta_{3}\right) \sin r_{o}{ }^{-1} t-0.5 \mu^{-2} \Gamma^{2} \sin r_{o}{ }^{-1} t\right]\right\} \\
& +\cdots \text {, } \\
& \begin{aligned}
x_{2}\left(1-\mu^{-2} e_{1} v\right)= & \left(\gamma_{o}^{\prime \prime}\right)^{-1}\left[\left(M_{3}+\beta_{3}\right) \cos r_{o}^{-1} t\right. \\
& \left.-0.5 \mu^{-2} \Gamma^{2} \cos r_{o}^{-1} t\right]+\mu^{-2} e v-\mu^{-2} v \\
& \cdot\left[\frac{x_{o}{ }^{\prime} \gamma_{o}^{\prime \prime}}{1-a}+e_{1}\left(M_{3}+\beta_{3}\right) \cos r_{o}^{-1} t\right]+\cdots
\end{aligned} \\
& \dot{x}_{2}\left(1-\mu^{-2} e_{1} v\right)=\left(\gamma_{o}{ }^{\prime \prime}\right)^{-1}\left[-\left(M_{3}+\beta_{3}\right) \sin r_{o}{ }^{-1} t\right. \\
& \left.+0.5 \mu^{-2} \Gamma^{2} \sin r_{o}^{-1} t\right]+\mu^{-2} v e_{1} \\
& \cdot\left(M_{3}+\beta_{3}\right) \sin r_{o}{ }^{-1} t+. \cdots
\end{aligned}
$$

Let the step $i=0: 300$ and $t=i T / 300$, where $T$ is the maximum value of the variable $t$. Let us introduce the following data:

$$
\begin{gathered}
A=10, \\
B=15, \\
C=20, \\
x_{0}=4, \\
y_{0}=7, \\
z_{0}=8, \\
M=600, \\
\gamma_{0}^{\prime \prime}=0.99, \\
r_{0}=0.000001, \\
T=21.76559 .
\end{gathered}
$$

Let the body parameters be

$$
\begin{gathered}
A_{1}=0.5, \\
B_{1}=-0.6666667, \\
C_{1}=0.25, \\
\omega=\sqrt{3} / 3, \\
\ell=11.357820, \\
x_{0}^{\prime}=0.3521804, \\
y_{0}^{\prime}=0.6163157, \\
z_{0}^{\prime}=0.7043607, \\
g=1.020000 E-04, \\
c=0.1864267395, \\
\varepsilon=5.272944 E 05, \\
a=0.5, \\
b=0.75, \\
v=2, \\
v_{1}=6, \\
e=-4, \\
e_{1}=-6.4, \\
e_{2}=-2.4 .
\end{gathered}
$$

Introduce the following computerized symbols:

$$
\frac{d x_{2}}{d t}=y, \quad x_{2}=J
$$

Using the above data and a computer program, we obtain the analytical solutions in the graphs (see Figures 1 and 2).

4.2. The Numerical Solutions. Using (18), we rewrite the system (1) in the form

$$
\begin{gathered}
\ddot{x}_{1}=-\omega^{2} x_{1}+\mu^{-2} F\left(x_{1}, J, \dot{x}_{1}, y, \mu^{-1}\right), \\
\dot{y}=-J+\mu^{-2} \Phi\left(x_{1}, J, \dot{x}_{1}, y, \mu^{-1}\right) .
\end{gathered}
$$

Using the fourth-order Runge-Kutta method [12] through a computer program and the data (16) and (17) with the same initial values of the analytical solutions, we obtain the numerical solutions in the graphs (see Figures 3 and 4). For checking the accuracy of both solutions, we draw the graphs (see Figures 5 and 6). We find agreement between the analytical and numerical solutions which satisfied the excellent results for the analytical and numerical techniques. The smooth simple curves obtained (see Figures 7-12) show that the obtained solutions are stable $[13,14]$. 


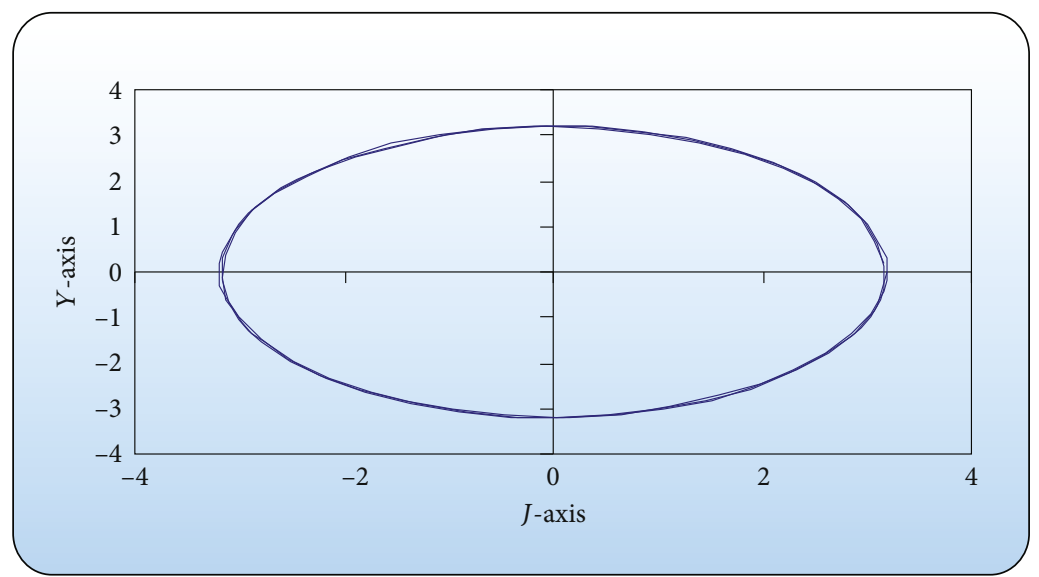

FIGURE 10: The stability of the analytical solutions $y=\dot{x}_{2}$ and $J=x_{2}$.

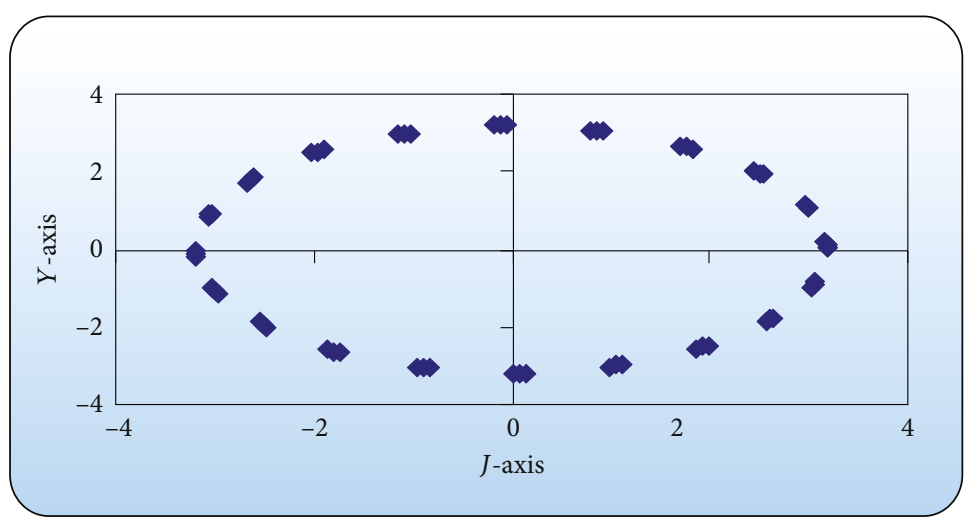

FIGURE 11: The stability of the numerical solutions $y=\dot{x}_{2}$ and $J=x_{2}$.

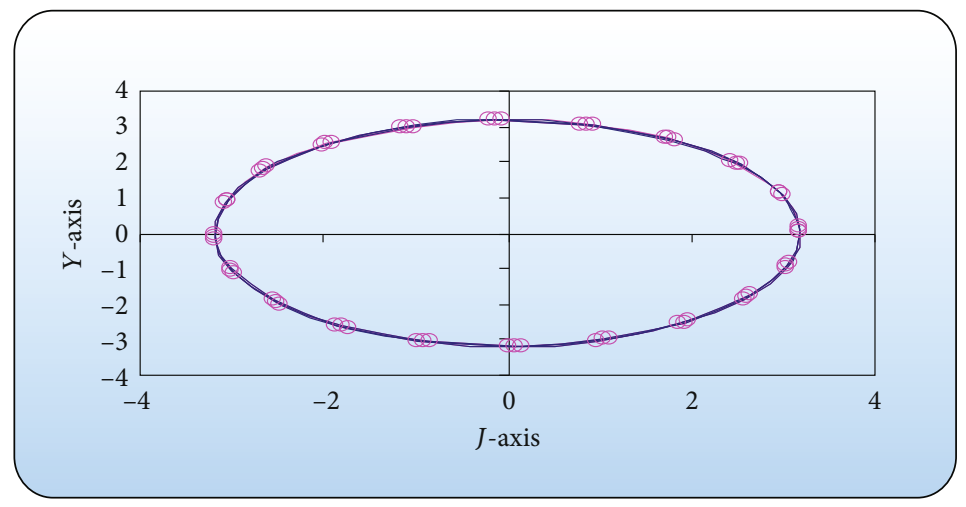

Figure 12: The stability of the analytical and numerical solutions $y=\dot{x}_{2}$ and $J=x_{2}$.

\section{Conclusions}

In this section, we conclude that the problem of the motion of a rigid body in a uniform gravity field with an irrational value of the natural frequency which is excluded from the previous works [1-3] is considered. The equations of motion and their first integrals in the presence of new conditions of motion are obtained and reduced to a semilinear autonomous system of two degrees of freedom and one first integral. We assumed a large parameter $\mu$ that is achieved to be inversely proportional to the angular velocity component $r_{\mathrm{o}}$ which is sup- posed to be sufficiently small. Under this assumption, the well-known Poincare method [15] cannot solve this problem because we cannot achieve the small parameter which must be proportional to a sufficiently high angular velocity component $r_{o} \rightarrow \infty$. Therefore, we solve the problem with the large parameter technique. The advantages of this method are as follows: using small energy at the initial moment instead of high energy, obtaining a slow gyroscopic motion instead of fast gyroscopic motion, and giving analytical and numerical solutions in a new domain of the motion $f\left(r_{o} \rightarrow 0, \mu \rightarrow \infty, t\right)$. Also, the correction of the period for these solutions is obtained 
in the new domain. The geometric interpretation of motion is attained. When $\mu \rightarrow \infty$, we obtain from Equation (17) the cases of regular permutation and pure rotation. Applying the numerical fourth-order Runge-Kutta method [12] through a computer program, we find the numerical solutions for this problem. Another computer program is carried out on the analytical obtained solutions using the large parameter technique and assuming the same initial values and data (16) and (17). We get the numerical results for both solutions and their graphical representations. The agreement of results through the graphical representations shows the advantage of both techniques for obtaining the high accuracy of the obtained solutions. This problem has many applications in aerospace sciences and technologies because of the wide use of gyros in these fields [16]. The procedures used here are useful for solving sophisticated problems such as [17] in a new domain of the considered parameters. This can be done by reflecting the problem parameters. In shaa Allah in next papers, we study the remaining singular cases of the natural frequency values to complete the solutions for the problem up to the third approximation.

\section{Data Availability}

Data sharing is not applicable to this article as no datasets were generated or analyzed during the current study.

\section{Conflicts of Interest}

The author declares that he has no competing interests.

\section{References}

[1] A. I. Ismail, "Solving a problem of rotary motion for a heavy solid using the large parameter method," Advances in Astronomy, vol. 2020, Article ID 2764867, 7 pages, 2020.

[2] A. I. Ismail, "Applying the large parameter technique for solving a slow rotary motion of a disc about a fixed point," International Journal of Aerospace Engineering, vol. 2020, Article ID 8854136, 7 pages, 2020.

[3] A. I. Ismail, "The slow spinning motion of a rigid body in Newtonian field and external torque," Advances in Astronomy, vol. 2020, Article ID 6629183, 12 pages, 2020.

[4] G. A. Sahli, "The motion of a rigid body in the presence of a gyrostatic momentum in cases of $\mathrm{l}_{3}$ and $\mathrm{l}_{3}=0$," Arab Journal of Sciences and Research Publishing, vol. 3, p. 1, 2019.

[5] M. Ehud, Nonlinear Physics of Ecosystems, Taylor \& Francis, Abingdon, England, 1st edition, 2019.

[6] A. V. Borisov and I. S. Mamaev, Rigid Body Dynamics, Higher Education Press and Walter de Gruyter Gmbh, China, 2018.

[7] T. S. Amer and I. M. Abady, "Solutions of Euler's dynamic equations for the motion of a rigid body," Journal of Aerospace Engineering, vol. 30, no. 4, p. 04017021, 2017.

[8] T. S. Amer, "The rotational motion of the electromagnetic symmetric rigid body," Applied Mathematics \& Information Sciences, vol. 10, no. 4, pp. 1453-1464, 2016.

[9] F. L. Chernousko, L. D. Akulenko, and D. D. Leshchenko, "Evolution of motions of a rigid body about its center of mass," Springer, Cham, 1st edition, 2017.
[10] A. A. Elmandouh, "New integrable problems in the dynamics of particle and rigid body," Acta Mechanica, vol. 226, pp. 3749-3762, 2015.

[11] A. A. Elmandouh, "New integrable problems in rigid body dynamics with quartic integrals," Acta Mechanica, vol. 226, no. 8, pp. 2461-2472, 2015.

[12] C. T. Wu, L. Wang, B. Bonello, L. Ling, N. Ma, and M. A. Schweitzer, "Advanced mesh-based and particle-based numerical methods for engineering and applied mathematics problems," Mathematical Problems in Engineering, vol. 2017, Article ID 1273017, 2 pages, 2017.

[13] W. S. Amer, "On the harmonic oscillations for the motion of a dynamical system," Journal Advances in Physics, vol. 13, no. 2, pp. 4657-4670, 2017.

[14] M. Iñarrea, V. Lanchares, A. I. Pascual, and A. Elipe, "Stability of the permanent rotations of an asymmetric gyrostat in a uniform Newtonian field," Applied Mathematics and Computation, vol. 293, pp. 404-415, 2017.

[15] M. P. Kharlamov and H. M. Yehia, "Separation of variables in one case of motion of a gyrostat acted upon by gravity and magnetic fields," Egyptian Journal of Basic and Applied Sciences, vol. 2, no. 3, pp. 236-242, 2019.

[16] V. S. Aslanov, Rigid Body Dynamics for Space Applications, Butter-Worth Heinemann, Oxford, 2017.

[17] M. M. Bhatti, R. Ellahi, A. Zeeshan, M. Marin, and N. Ijaz, "Numerical study of heat transfer and Hall current impact on peristaltic propulsion of particle-fluid suspension with compliant wall properties," Modern Physics Letters B, vol. 33, no. 35, article 1950439, 2019. 